\title{
Les évangéliques, l'Honorable Compagnie des Indes Orientales et la christianisation du sous-continent indien au tournant du XIX siècle
}

Evangelicals, the Honourable East India Company and the Christianisation of the Indian Sub-continent at the Turn of the Nineteenth Century

\section{Evelyne Hanquart-Turner}

\section{OpenEdition \\ Journals}

Édition électronique

URL : http://journals.openedition.org/rfcb/3620

DOI : $10.4000 /$ rfcb.3620

ISSN : 2429-4373

Éditeur

CRECIB - Centre de recherche et d'études en civilisation britannique

Édition imprimée

Date de publication : 1 mars 2013

ISBN : 2-911580-37-0

ISSN : 0248-9015

Référence électronique

Evelyne Hanquart-Turner, « Les évangéliques, I'Honorable Compagnie des Indes Orientales et la christianisation du sous-continent indien au tournant du XIXe siècle », Revue Française de Civilisation Britannique [En ligne], XVIII-1 | 2013, mis en ligne le 01 mars 2013, consulté le 22 mars 2020. URL http://journals.openedition.org/rfcb/3620 ; DOI : https://doi.org/10.4000/rfcb.3620

Ce document a été généré automatiquement le 22 mars 2020.

\section{(i) $९$

Revue française de civilisation britannique est mis à disposition selon les termes de la licence Creative Commons Attribution - Pas d'Utilisation Commerciale - Pas de Modification 4.0 International. 


\title{
Les évangéliques, l'Honorable Compagnie des Indes Orientales et la christianisation du sous- continent indien au tournant $\mathrm{du}$ $\mathrm{XIX}^{\mathrm{e}}$ siècle
}

\author{
Evangelicals, the Honourable East India Company and the Christianisation of the \\ Indian Sub-continent at the Turn of the Nineteenth Century
}

\section{Evelyne Hanquart-Turner}

$1 \mathrm{Si}$, selon l'expression reprise par Keki Daruwalla, les premières expéditions portugaises vers les Indes étaient parties " pour le poivre et le Christ $»^{1}$, les marchands londoniens qui fondèrent la Compagnie des Indes Orientales (East India Company) en 1600, obtenant charte royale et monopole commercial, n'étaient, eux, directement intéressés que par le poivre, les épices et autres denrées. L'illusion de retrouver aux Indes le légendaire royaume du Prêtre Jean était alors abandonnée depuis longtemps. On savait que les seuls chrétiens des Indes étaient ceux de l'Église syriaque du Kerala et la minorité de catholiques romains convertis par les Portugais ou les Français. La Compagnie était initialement hostile à la venue de missionnaires sur ses territoires, car elle y voyait non sans raison, comme le prouvera la situation dès l'époque de Lord Bentinck (1828-1835), puis de Lord Dalhousie (1848-1856) - un facteur de troubles et de tensions avec les populations locales, hindoues ou musulmanes, tensions qui pourraient nuire au commerce. Elle était alors bien davantage préoccupée de poursuivre, plutôt que celle de Dieu, l'œuvre de César, et de satisfaire les intérêts du royaume, de l'Institution et de ses actionnaires.

2 Les besoins spirituels des employés de la Compagnie cantonnés dans les trois comptoirs de Calcutta, Madras et Bombay étaient pris en charge par des aumôniers engagés par ses soins et arrivés avec eux de la métropole ${ }^{2}$. Il était hors de question que la religion chrétienne se manifestât dans la vie publique à l'extérieur de ses établissements et des 
lieux de résidence des Européens. Il n'y avait donc pas alors, pour la Compagnie, de « question religieuse » à considérer.

3 Avec le renouveau spirituel, anglican et dissident, qui transforme l'Angleterre pendant les dernières décennies du XVIII ${ }^{e}$ siècle et atteint tout particulièrement les classes dont font partie à la fois les actionnaires, les dirigeants et les employés de la Compagnie, la situation évolue dramatiquement. Cette classe en pleine ascension sociale voit en effet dans l'élan religieux britannique non seulement une réaction opportune contre le laxisme des mœurs, mais un mouvement d'union patriotique salutaire contre les influences néfastes de la pensée des Lumières, de la pensée française en particulier :

[The philosophes] exhibit a most disgusting prostitution of the intellect, a complete dereliction of moral principle and feeling, and a cool deliberate hostility to the quietude and best interests of their species. [...] The sincere and zealous friends of the Gospel throughout the kingdom, impressed with the solemn peculiarities of the time, yielded to the call. ${ }^{3}$

4 L'influence évangélique s'étend donc progressivement au sein de cette bourgeoisie, au point d'être perçue par certains comme une force quelque peu menaçante, comme le montre cet extrait du Monthly Repository unitarien de 1808 :

The Evangelical party (the most numerous association of men for a particular object in the kingdom) have been of late drawn into closer union. They have no mean number of supporters in the bosom of the Church of England, and they may safely reckon upon nine-tenths of the persons not in communion with the established church. Even the Quakers have shewn a leaning to the Evangelical religionists. In fact, the liberal part of the clergy, with their adherents and supporters amongst the body of the people, and the Unitarian dissenters, are the only classes that oppose any obstacle to their progress. They have invaded the Navy, they thrive at the Bank, they bear sway at the India House, they count several votes in Parliament, and they have got a footing in the Royal Palace. Their activity is incredible. ${ }^{4}$

Or, pour ce qui est de la Compagnie, Bernard Cohn a montré à quel point ses membres et ses employés étaient liés, et appartenaient à un milieu étroitement homogène :

To summarize, the directors of the East India Company, and by extension the Civil Service were recruited from a very restricted group in English society centered essentially in London and drawn from banking and commercial families and landed groups in Scotland and the southeast of England. Cultural and economic ties of these groups were very much buttressed by ties of descent and affinity. ${ }^{5}$

Le réseau d'influence de ces familles, pour la plupart de tendance évangélique, s'avère déterminant dans l'essor commercial, financier et politique autant que dans le développement religieux en métropole comme aux Indes. Le cercle des Wilberforce, Thornton, Grant, Stephen, alliés et amis, est exemplaire pour notre propos.

$7 \mathrm{Si}$ « [l]'interdépendance entre la Cité et Westminster a été non seulement le succès final dans les guerres de la Révolution et de l'Empire " ${ }^{6}$, l'influence morale des évangéliques, en nombre dans la Cité et à Westminster, tout comme dans la Compagnie des Indes ${ }^{7}$, va être déterminante pour les deux grandes causes que sont l'abolition de la traite et de l'esclavage et la conversion des hérétiques et des païens ${ }^{8}$. Avec la troisième génération des Thornton, les liens avec la Très Honorable Compagnie se renforcent. Chacun d'entre eux, comme leur cousin Wilberforce, y possède plus de 2000 livres d'actions, ce qui les rend ainsi éligibles au Conseil d'administration (Court of Directors). Il est toutefois évident que ces banquiers, Robert et Samuel Thornton, n'auraient pu y atteindre les niveaux de responsabilité qui furent les leurs sans le poids de la colossale fortune de leur père, John, et de leur parentèle, en tant qu'actionnaires. John avait consacré une 
part importante de sa fortune à l'hôpital militaire de Dulwich, à la Bible Society, à la Society for Promoting Christian Knowledge, et à l'achat d'advowsons ${ }^{9}$ destinés à promouvoir la carrière de jeunes ecclésiastiques de tendance évangélique. Son influence s'étendait donc aux divers cercles de l'Église anglicane et aux compagnies négociant avec les éventuelles terres de mission, ses trois fils, Samuel, Robert et Henry œuvrant de même, tout comme William Wilberforce, ou leurs amis et voisins, Sir James Mackintosh, Charles Grant, Zachary Macaulay, Lord Teignmouth, James Stephen, et John Venn, curé de Clapham. C'est ainsi qu'on les compte en 1799 parmi les membres fondateurs de la Church Missionary Society (CMS), de la Religious Tracts Society (RTS), créée par Hannah More la même année, puis de la British and Foreign Bible Society (BFBS), en 1804.

\section{L'Inde, terre de mission?}

8 Si le but de la RTS était l'éducation chrétienne des classes populaires anglaises, la CMS et la BFBS étaient davantage tournées vers l'extérieur du pays, d'autant plus que la faction évangélique n'avait pas réussi à se faire entendre au Parlement lors du renouvellement de la charte en $1793^{10}$. Elle n'avait pas pu forcer la main au Conseil d'administration pour ouvrir l'accès des territoires de la Compagnie aux missionnaires. En effet, à mesure que le désir de répandre les idéaux de l'Évangile et de promouvoir leur mise en œuvre à travers des organismes tels que la Society for Promoting Christian Knowledge s'était intensifié en Grande-Bretagne et que s'était renforcé le poids financier et politique des évangéliques, ces derniers avaient manifesté une volonté grandissante de faire prévaloir leurs vues au Parlement comme au Conseil d'administration de la Compagnie grâce à de fortes personnalités telles que Charles Grant et William Wilberforce. En vain avaient-ils poussé à l'insertion dans la charte de nouvelles clauses - les «clauses pieuses» (pious clauses) - qui auraient ouvert la voie des Indes aux missionnaires - de préférence anglicans - pour éclairer les idolâtres et les hérétiques, leur faire découvrir les vertus chrétiennes et, ainsi, les amener vers la vraie foi. Par ces clauses, la Compagnie aurait d'abord dû reconnaître sa responsabilité de promouvoir " le progrès moral et religieux de l'Inde » et, à cet effet, de fournir « des hommes qualifiés pour y être maîtres d'école, missionnaires et autres. ${ }^{11}$ Cet échec ne découragea cependant pas l'effort de ceux que leurs collègues parlementaires appelaient, non sans dérision, « les saints ». En effet, ces derniers s'employèrent, par la fondation de la CMS et de la BFBS, comme par celle de leur périodique, The Christian Observer (1802), à mobiliser la nation tout entière pour le prochain renouvellement de la charte prévu en 1813.

Ceci ne signifiait pas l'absence de missionnaires aux Indes, mais qu'ils opéraient seulement dans les États princiers et les comptoirs d'autres nations européennes parfois, plus ou moins clandestinement, depuis ces territoires. Les deux meilleurs exemples en étaient les comptoirs danois de Serampore (au Bengale) et de Tranquebar (au Tamil Nadu), où étaient implantés des baptistes britanniques tels que Joshuah et Hannah Marshman, William Ward et, surtout, William Carey, ou des luthériens, souvent d'origine allemande, tel Christian Frederick Schwartz. À Serampore, sous la direction de Carey et de sa petite équipe de traducteurs, une Bible en bengali est imprimée dès 1800 , suivie d'une version en sanscrit et d'autres traductions des livres des deux Testaments dans de multiples langues vernaculaires. Étant donné sa situation géographique proche de Calcutta, la mission de Serampore n'était pas sans gêner la politique religieuse de la Compagnie. À Tranquebar, les presses de la mission 
produisirent dès le milieu du XVIII ${ }^{e}$ siècle une version tamoule de la Bible due essentiellement aux travaux de Schwartz, suivie d'une traduction en hindoustani, lingua franca sur de vastes étendues du sous-continent. Au tournant du XIX ${ }^{\mathrm{e}}$ siècle, l'influence de cette mission s'étendait jusqu'à Madras et à Tanjore. La situation ne pouvait guère satisfaire la Compagnie, que ce soit les responsables londoniens ou les chapelains anglicans sur le terrain, qui voyaient leur politique de non-ingérence menacée, ni les évangéliques, qui assistaient, impuissants, aux avancées des missionnaires, protestants certes, mais dissidents.

10 Ainsi, pendant les deux décennies suivantes, la mobilisation des évangéliques entrâne la création de multiples branches des Sociétés pour la Propagation du Christianisme et de la Bible au Royaume-Uni comme aux Indes, ce maillage du territoire ayant le triple effet de sensibiliser l'opinion à la question de l'évangélisation, de rassembler différentes catégories sociales dans un même effort à un moment de danger national, et de collecter des fonds importants sur une durée assez longue pour les rendre considérables. Comme le veut la tradition évangélique, l'accent est mis sur la lecture des livres saints et le salut par les œuvres, ce qui facilite le rassemblement de participants de sensibilités religieuses différentes.

11 La controverse sur l'ouverture aux missions des territoires toujours croissants de la Compagnie n'est donc pas close par le vote de la charte de 1793. Les vingt années suivantes voient la question religieuse occuper une place significative pour ces marchands devenus gouvernants ${ }^{12}$, que ce soit dans les débats des assemblées générales des actionnaires (Court of Proprietors) ou sous forme de lettres ouvertes et de pamphlets. À la lumière des événements qui suivront, quelques décennies plus tard, ce débat prend un tour particulièrement intéressant lorsqu'en 1807, Thomas Twining, cadre de la Compagnie, 'senior merchant', et fils de l'un des membres du Conseil d'administration (Court of Directors), exprime ses inquiétudes et ses réserves dans une lettre ouverte au président, le très évangélique Edward Parry ${ }^{13}$. Après la mutinerie de Vellore l'année précédente ${ }^{14}$, Twining, qui a alors passé une douzaine d'années aux Indes, s'inquiète de la diffusion des textes chrétiens traduits et, surtout, de ce qu'il considère comme l'intrusion illégale des missionnaires sur le territoire de la Compagnie, prédisant les pires conséquences pour la paix publique. Cette longue missive est particulièrement intéressante, parce qu'elle émane d'un homme qui connaît bien l'Inde, mais aussi parce qu'elle cite abondamment des textes écrits par ces missionnaires, textes révélateurs de leurs attitudes vis-à-vis de la culture et de la religion locales. On y trouve en particulier des phrases incendiaires de la part d'hommes aussi influents que William Carey, William Ward (de la mission baptiste), et que David Brown et Claudius Buchanan - ces deux derniers étant docteurs en théologie et, respectivement, directeur et vicedirecteur ducollège de la Compagnie à Fort William (Calcutta) ${ }^{15}$. Ces savants ecclésiastiques directement impliqués dans l'œuvre de traduction biblique font part aux responsables de la BFBS de leur total mépris des indigènes et de la civilisation locale, et ne cachent pas leur dessein missionnaire au-delà de la simple traduction et diffusion de textes chrétiens :

You will sometimes hear it said that the Hindoos are a mild and passive people. They have apathy rather than mildness; their hebetude of mind is, perhaps, their chief negative virtue. They are a race of men of weak bodily frame, and they have a mind conformed to it, timid, abject in the extreme. They are passive enough to receive any vicious impression [...] What is chiefly worthy of notice, we can approach them in no other way than by means of our religion. ${ }^{16}$ 
12 Twining s'inquiète de cette ferveur prosélyte, d'autant plus que Brown et Buchanan sont aumôniers de la Compagnie, et agissent donc explicitement en contradiction avec la politique de leur employeur. Ces citations, sans aucun doute choisies avec soin, sont d'une grande violence : elles préconisent la conversion forcée des indigènes pour, selon les auteurs, faciliter le gouvernement de la Compagnie et amener à la vraie foi païens et hérétiques pour la plus grande gloire de Dieu et le bénéfice moral des gouvernants et de leurs sujets ${ }^{17}:$ 'A wise policy seems to demand that we should use every means of coercing this contemptuous spirit [towards Christianity] of our native subjects'. La lettre de Twining déclenche une polémique avec John Owen, secrétaire de la BFBS, et Andrew Fuller, celui de la Baptist Missionary Society, lui-même missionnaire, d'autant plus agressif que son action est directement attaquée. Le second point que soulève Twining est celui des liens étroits existant entre les auteurs de ces propos et de ces comportements et les responsables de l'Honorable Compagnie et du Comité de Contrôle parlementaire, mettant ainsi en évidence une forme de collusion entre eux, puisqu'il ne peut plus y avoir de réelle volonté politique de limiter les actions des missionnaires. Cette guerre des pamphlets anime les assemblées générales de la Compagnie pendant plus d'un an.

Ces débats qui mettent les questions religieuses au premier plan de l'action administrative et politique de la Compagnie sont révélateurs de leur importance croissante dans la politique indienne au niveau de la Compagnie comme du Parlement, certains prédisant la chute du pouvoir britannique aux Indes si elles deviennent terre de mission, les autres défendant leur entreprise de diffusion des valeurs chrétiennes au sein de cultures jugées sans valeur, voire carrément immorales. Ce crescendo dans l'intérêt porté aux questions religieuses s'amplifie avec la perspective du renouvellement de la charte, d'autant plus que l'opinion publique en Angleterre comme en Inde a été alertée par une importante mobilisation dans les branches locales de la CMS et de la BFBS et par les campagnes de pétitions en faveur des missionnaires menées par des évangéliques influents tels que Granville Sharp ou William Wilberforce. C'est ainsi qu'on remarque Henry Martyn, fellow de St John's College, Cambridge, et aumônier de la Compagnie, qui prêche le jour de l'an 1811 devant les résidents britanniques de Calcutta en faveur de la BFBS. La collecte atteindra près de 10000 roupies $^{18}$.

\section{Ouverture aux missionnaires et création d'un évêché}

En 1813, alors que Robert Thornton est président de la Compagnie (mais également membre du Conseil d'administration de la BFBS et de la Chambre des Communes), la charte est renouvelée. Une nouvelle version des « clauses pieuses » est adoptée malgré l'opposition du président du Comité de Contrôle, Robert Dundas, membre pourtant fort influent de la Compagnie ${ }^{19}$. Cette dernière se voit imposer de promouvoir, moyennant un financement de 10000 livres par an, "l'éducation pratique et l'édification morale et religieuse de la population qui se trouve sur son territoire ${ }^{20}$. La clause XXIII rend légalement obligatoire pour la Compagnie d'étendre à ses sujets indigènes sa responsabilité éducative, morale et religieuse. À ceci s'ajoute un texte de loi créant un évêché anglican sis à Calcutta, mais couvrant l'Inde entière et Ceylan. La religion chrétienne, dans sa version anglicane, prend donc officiellement pied sur le souscontinent; elle fait désormais partie des institutions britanniques dont le pouvoir 
s'étend au fil des quatre décennies suivantes pour atteindre les trois cinquièmes de l'Inde avant la Révolte des Cipayes, qui entraînera la disparition de la Compagnie.

Cette ouverture des Indes à l'évangélisation ne signifie pas cependant la fin de la question religieuse. Elle va simplement prendre une autre forme : celle d'une rivalité plus ou moins larvée entre missionnaires et Église officielle. Il est intéressant de considérer l'opinion de l'abbé Dubois sur la question de la christianisation des Indes. Ce prêtre jésuite, qui avait alors passé une trentaine d'années comme missionnaire dans l'État de Mysore, déclare dans des lettres ouvertes à des amis britanniques sa conviction de l'impossibilité de la tâche. À ses yeux, il est illusoire d'attendre une conversion massive et durable des hindous, et l'œuvre de traduction des textes chrétiens n'y change rien, bien au contraire, étant donné sa mauvaise qualité certaines de ces traductions confinent souvent au ridicule - qui, tout comme le comportement général des Européens, contribue à déconsidérer le christianisme :

Christianity became more and more an object of contempt and aversion in proportion as the European manners became better known to the Hindoos [...] The very name of Christian carries along with it the stain of infamy, and the proposal alone to become a convert to Christianity is considered by every well-bred Hindoo as a very serious insult, which is instantly resented. ${ }^{21}$

Une autre difficulté qui fait débat, en écho aux rivalités métropolitaines de l'époque, est cette fois liée aux relations entre l'évêché et la CMS. Le premier évêque de Calcutta, Thomas Farnshaw Middleton, de tendance 'high Church', considérait que ses lettres patentes ne s'étendaient pas aux missionnaires, ni à l'ordination du clergé indigène, mais limitaient sa juridiction aux aumôniers de la Compagnie, au contrôle de leurs activités, et à l'octroi - ou non - de la 'licence to preach' ${ }^{22}$. Lorsqu'il meurt à la tâche après avoir mis sur pied et géré son énorme diocèse pendant sept ans, il a pour successeur Reginald Heber (1783-1826), évangélique proche de la secte de Clapham et de la CMS, qui a une vision de son rôle quelque peu différente. Ce dernier choisit comme archidiacre pour le seconder le non moins évangélique Daniel Corrie, protégé de Charles Simeon et futur évêque de Madras, et se montre donc très coopératif avec les organisations missionnaires, accordant à certains de leurs membres la licence to preach'. Il ordonne même le premier prêtre indigène. À sa mort brutale en $1826^{23}$, la SPCK et la CMS unissent leurs efforts, mais en vain, pour obtenir du gouvernement la division du diocèse en trois entités distinctes ${ }^{24}$.

Avec la création d'un évêché et l'arrivée de missionnaires anglicans "officiels", l'influence évangélique en Inde s'étend rapidement. Bon nombre de ces hommes d'Église, tels Henry Martyn ou Daniel Corrie, ont en effet été formés à Cambridge, où ils ont subi l'influence de Charles Simeon, charismatique fellow de King's College et curé de Holy Trinity, ou d'Isaac Milner, Master de Queen's, tous deux promoteurs de la pensée évangélique ${ }^{25}$.

$18 \mathrm{Au}$ cours de leurs visites pastorales qui les entraînent pendant des mois à travers l'Inde entière, Middleton, Heber et leur suite perçoivent l'ampleur de la tâche à accomplir, et font le point sur la situation du christianisme indien. Les moyens manquent aux missions pour atteindre la population indigène, et leur action se trouve souvent limitée aux Eurasiens et aux Indiens précédemment convertis ('native Christians'). Middleton et Heber déplorent le caractère superficiel de la foi et de la pratique religieuse chez la plupart de leurs compatriotes résidant dans les comptoirs, où le nombre d'aumôniers est insuffisant. Ils constatent avec effroi l'état des croyances religieuses des catholiques 
romains du sud de l'Inde, guère meilleur, selon eux, que celui des païens hindous en matière de morale et d'idolâtrie ${ }^{26}$. Ils s'intéressent en revanche de près, et avec bienveillance, à l'Église syriaque du Kerala, la plus ancienne des Indes. Il s'établit même un dialogue entre l'évêque et le patriarche dans le but de déboucher, selon le désir exprimé par ce dernier, semble-t-il, sur une version de la liturgie anglicane en syriaque, langue savante, et en malayam, langue vernaculaire de cette communauté. Heber manifeste aussi une certaine bienveillance envers les missionnaires luthériens et leurs homologues baptistes durant ces visites, mais cette attitude œcuménique reste marquée par une certaine réserve, probablement liée à sa position officielle plus qu'à son inclination personnelle, si l'on en juge par les parties publiées de sa correspondance; l'orthodoxie anglicane ne pouvait totalement fermer les yeux sur les déviances dissidentes, même si luthériens et baptistes avaient jusque-là préparé le terrain.

19 Pour Middleton comme pour Heber, il s'agit avant tout de donner des bases et une implantation solides à l'Église anglicane des Indes pour lui assurer une vigueur et une efficacité pérennes. Dès 1818, Middleton avait fait part d'un projet en ce sens à la SPGFP ${ }^{27}$. Il avait l'intention de fonder un collège de missionnaires aux environs de Calcutta dans le quadruple but :

1. Of instructing Native and other Christian youths in the doctrine and discipline of the Church, in order to their becoming preachers, catechists and school teachers.

2. For teaching the elements of useful knowledge and the English language to Musulmans or Hindoos having no object in such attainments beyond secular advantage.

3. For translating the Scriptures, the liturgy, and moral and religious tracts.

4. For the reception of English Missionaries to be sent out by the Society, on their first arrival. 28

Grâce aux 5000 livres accordées par la Société pour l'achat du terrain et la construction des bâtiments, et d'autres financements venus de la SPCK et de la BFBS, Mission College, qui devient ensuite Bishop's College, voit le jour en 1824, après la mort de son fondateur. L'arrivée du très évangélique Heber ne change pas la destinée du Collège, et ses successeurs - Daniel Wilson, 'the evangelical of evangelicals' ${ }^{29}$, en particulier soutiennent l'action du Collège malgré leurs difficultés récurrentes avec la CMS et les missionnaires, auxquels ils reprochent souvent la piètre qualité de leur enseignement, qu'il soit religieux ou profane.

21 La situation se complique encore en 1839 lorsque l'évêque se trouve confronté à la nomination d'un nouveau directeur, Arthur Wallis Street, de tendance tractarienne. Wilson s'inquiète alors que la formation des futurs missionnaires anglicans puisse être confiée à un professeur proche de Manning et recommandé par Newman. Pour rétablir l'équilibre à ses yeux, il appuie en 1843 - comme d'ailleurs Henry Venn, héritier de Clapham et secrétaire de la CMS - la nomination du second professeur, George Weidermann, un évangélique de St Catharine's College, Cambridge.

\section{La controverse de la 'pilgrim tax'}

Sur un plan plus général, toutefois, Wilson n'a plus à s'inquiéter des tractariens. Il n'a rien à craindre du gouvernement de Bentinck inspiré par Thomas Babington Macaulay, cet autre héritier de Clapham; l'évangélisme, comme l'utilitarisme, imprègne toute la 
politique éducative, culturelle et sociale dans l'Inde britannique; la lutte contre les coutumes hindoues les plus obscurantistes s'accélère. L'attitude bienveillante de la Compagnie envers les rites dénoncée par certains dès le début du siècle rencontre désormais l'opposition des missionnaires, bien sûr, mais aussi des actionnaires, tel John Poynder, qui mène depuis des années une lutte incessante dans les assemblées générales pour l'abolition du sati ${ }^{30}$, l'une des premières mesures d'ailleurs prises par Bentinck. En septembre 1830, il prononce devant l'assemblée générale un discours publié ensuite sous forme de pamphlet dans lequel il attaque la Compagnie en tant que gouvernant pour son comportement face aux pèlerinages hindous et aux pratiques sacrificielles qui les accompagnent. La controverse venait du fait que la Compagnie, qui était désormais l'autorité politique et administrative en charge des temples hindous, devait en garantir l'entretien, comme la sécurité des milliers de pèlerins qui s'y rendaient. Pour couvrir ces frais, la tradition séculaire indienne était de percevoir une taxe (pilgrim tax) de tous ceux qui venaient faire leurs dévotions. Pour assurer la police des grands pèlerinages, la Compagnie utilisait les cipayes de son armée. Avec Poynder, les opposants considèrent qu'ainsi elle encourage activement l'idolâtrie et la superstition, d'une part en rendant plus facile et plus sûr l'accès aux temples, d'autre part en finançant leur entretien, celui des prêtres et de leurs acolytes, et en fermant les yeux sur toutes les formes de rituels - en particulier orgastiques et sacrificiels, de torture et de mutilation - que s'infligent certains dévots hindous. Selon Poynder, ce comportement de la Compagnie va à l'encontre des stipulations de la charte concernant le progrès moral et religieux des Indes. De plus, l'immoralité de cette situation scandaleuse est accrue par le fait qu'une compagnie chrétienne soutenue par un État chrétien tire des milliers de livres de profit au bénéfice involontaire d'actionnaires révoltés par les pratiques idolâtres païennes ${ }^{31}$. Poynder propose donc le vote d'une motion demandant l'intervention du Conseil d'administration auprès du gouvernement des Indes « pour enfin laver un empire chrétien d'une telle souillure ». Sitôt la motion rejetée par les actionnaires, un comité parlementaire est créé pour enquêter sur cette question. John Stuart Mill, secrétaire de la Compagnie, est en faveur du maintien de la taxe, mais d'autres, qui ont une expérience directe de la vie indienne, témoignent en des termes comparables à ceux de Poynder :

But, the muscle in the Evangelical attack came from the younger Charles Grant who, from his position as President of the Board of Control, manipulated Company policy. He persuaded the Board that they should override all objections from the Court of Directors and demand the abolition of the Pilgrim Tax as well as all British interference in Temple management. ${ }^{32}$

Ces ordres sont bien transmis dès février suivant, mais, comme l'administration de la Compagnie n'y est en fait guère favorable, il faudra quelque temps pour les voir appliquer ! En fait, c'est le mémoire sur l'éducation (1835) et le code pénal proposé par Macaulay - finalement appliqué après la Révolte des Cipayes - qui donnent au gouvernement de la Compagnie une orientation résolument évangélique, laquelle va influencer la formation des élites religieuses et laïques, et déterminer le sort de millions d'Indiens pour les décennies, voire le siècle à venir, qu'ils soient sensibles ou non à la parole de l'Évangile. 


\section{Conclusion}

$\mathrm{Si}$, au XVIII ${ }^{e}$ siècle, la politique religieuse de la Compagnie était donc déterminée par la question de l'introduction, ou non, de missionnaires sur son territoire et la tolérance bienveillante des coutumes locales, pour ne pas courir le risque de perturber la paix civile et, par conséquent, ses intérêts commerciaux, au tournant du XIX ${ }^{e}$ siècle, la montée en puissance de la religiosité britannique - anglicane ou méthodiste, en particulier - a rendu impossible le statu quo, et a forcé la main de ses dirigeants les plus réticents, conduisant à faire des Indes non seulement une terre de mission au sens habituel, mais aussi une province de l'Église établie d'Angleterre, c'est-à-dire une partie intégrante du système "constitutionnel » britannique ${ }^{33}$, où les valeurs éducatives et morales priment sur les principes théologiques. Dans les décennies qui suivent, le rôle des missionnaires s'oriente de plus en plus vers les questions sociales plutôt que vers la transmission du dogme, se focalisant surla formation des jeunes (en particulier des élites), sur l'instruction des femmes ('zenana missions'34) ou sur les œuvres de charité (surtout hospitalières), en accord avec l'inspiration évangélique dominante. On peut donc penser que le rôle des évangéliques dans le cadre de la Compagnie des Indes a davantage contribué à l'occidentalisation du sous-continent qu'à sa christianisation.

\section{NOTES}

1. Keki N. DARUWALLA, For Pepper and Christ, New Delhi: Penguin India, 2009.

2. Selon le East India Register de 1813 (cité dans Claudius BUCHANAN, Colonial Ecclesiastical Establishment : Being a Brief View of the State of the Colonies of Great Britain, and of her Asiatic Empire, in Respect to Religious Instruction, London: Cadell \& Davies, 1813, p. 145), la Compagnie comptait 35 aumôniers (dont 15 au Bengale, 13 sur le territoire de Madras, et 5 sur celui de Bombay), qui percevaient un salaire moyen de 800 livres par an.

3. William A. THOMSON \& William ORME, A Historical Sketch of the Translation and Circulation of the Scriptures: from the Earliest Period to the Present Time, Perth, 1815, pp. 96, 104.

4. R. ASPLAND (ed.), Monthly Repository of Theology and General Literature, 21 vols., London: Longman, Hurst, Rees \& Orme, 1806-1826, vol. 3, pp. 503-504. Cité dans Gareth ATKINS, 'Wilberforce and his Milieux: the Worlds of Anglican Evangelicalism c.1780-1830', thèse de doctorat de l'Université de Cambridge soutenue en 2009, p. 18.

5. Bernard S. COHN, 'Recruitment and Training of the British Civil Servants in India, 1600-1800', in Ralph BRAIBANTI et al. (eds.), Asian Bureaucratic Systems Emergent from the British Imperial Tradition, Durham, N.C.: Duke University Press, 1966, p. 110.

6. André GUILLAUME \& Marie-Claude ESPOSITO, Londres. Histoire d'une place financière, Paris : PUF, 1993, p. 6.

7. La Compagnie avait d'ailleurs grandement contribué au financement de ces guerres. Voir COHN, Ibid.

8. Selon Bernard S. Cohn, de 1784 à 1834,45 des 110 membres du Conseil d'administration siégeaient aussi aux Communes. 
9. E.M. FORSTER, Marianne Thornton, Évelyne HANQUART-TURNER (ed.), London: André Deutsch (Abinger edition), 2000. Voir aussi E. HANQUART-TURNER, « Pouvoir, commerce et religion. Les Thornton et le cercle d'influence de la Compagnie des Indes au tournant du XIX ${ }^{\mathrm{e}}$ siècle », in $\mathrm{E}$. HANQUART-TURNER (ed.), L'Empire et son double. Pouvoir et légitimité, Yaoundé: Nouvelles du Sud, 2003. En droit ecclésiastique anglican, un advowson est le droit que possédait le propriétaire d'un domaine, d'une seigneurie, de choisir le curé des paroisses dépendant de ses terres. Ce droit concernait aussi les paroisses urbaines ainsi dépendantes de propriétaires individuels ou institutionnels tels que les Collèges d'oxford ou de Cambridge, par exemple. Le choix était ensuite validé par l'évêque, qui nommait officiellement le prêtre concerné.

10. Ceci, malgré l'influent pamphlet de Charles Grant, Observations on the State of Society among the Asiatic Subjects of Great Britain, particularly with respect to morals and the means of improving it, qui avait lancé la campagne l'année précédente. Grant (1746-1823) avait vécu pendant vingt-deux ans au Bengale, où il avait travaillé comme employé civil, avant de devenir membre du Conseil d'administration, puis vice-directeur et directeur de la Compagnie à plusieurs reprises. Ses enfants le surnommaient d'ailleurs « le Directeur».

11. Cornelia WITZ, Religionpolitik in Britisch-Indien, 1793-1813. Christliches Sendungbewussten und Achtung Hinduistischer Tradition in Widerstreit, Stuttgart : Steiner Verlag, 1985.

12. Par un décret royal (firman) de 1764, le Grand Moghol avait confié l'administration, la justice et la perception des impôts du Bengale à la Très Honorable Compagnie.

13. Thomas Twining (1776-1861), second fils de Richard Twining (de la célèbre maison de thé), avait été envoyé aux Indes à l'âge de seize ans, et y avait servi comme employé civil.

14. Les soldats hindous et musulmans de la Compagnie s'y étaient révoltés contre l'interdiction qui venait de leur être faite de porter la barbe, leurs coiffures traditionnelles, et leurs signes de caste.

15. On y enseignait les langues vernaculaires aux jeunes employés civils avant de les envoyer sur le terrain. Buchanan et Ward étaient des protégés de Charles Grant, qui avait recommandé leur nomination.

16. Thomas TWINING, A Letter, to the Chairman of the East India Company: On the Danger of Interfering with the Religious Opinions of the Natives of India; and on the Views of the British and Foreign Bible Society, as Directed to India, $2^{\text {nd }}$ ed., London: J. Ridgway, 1807, p. 22.

17. Ibid., p. 21.

18. Henry MARTYN, Christian India, or an Appeal on Behalf of 900,000 Christians in India who want the Bible, Calcutta: P. Ferris, 1811. 10000 roupies semblent correspondre à peu près à 1000 livres de l'époque.

19. Robert Dundas (1776-1861), deuxième vicomte Melville, parent des Thornton par mariage, représentait aussi l'intérêt écossais au sein de la Compagnie.

20. East India Company Charter Act, 1813.

21. Jean-Antoine DUBOIS (abbé), Letters on the State of Christianity in India; in which the Conversion of the Hindoos is Considered as Impracticable, London: Longman, 1823, pp. 12, 14.

22. Timothy E. YATES, Venn and the Victorian Bishops abroad, Uppsala and London: Swedish Institute of Missionary Research, 1978, p. 24.

23. Il mourut d'hydrocution lors d'une visite pastorale à Trichonopoly, à des milliers de kilomètres de Calcutta.

24. Il faut attendre la charte de 1833 pour voir la création des diocèses de Madras et de Bombay.

25. Charles Simeon (1759-1836) était aussi un des membres fondateurs de la CMS, et conseiller de la Compagnie des Indes pour la nomination de ses aumôniers.

26. DUBOIS, op. cit., pp. 18-19, 23-24. Dans la première lettre, datée de 1815, l'abbé Dubois fait observer que le rituel catholique, avec ses processions, ses statues et son encens, plaît aux hindous, alors que le protestantisme, trop strict dans ses manifestations, ne leur parle pas. 
27. La Society for the Propagation of the Gospel in Foreign Parts avait initialement été fondée en 1701 par l'Église anglicane pour porter l'Évangile en Amérique et dans la Caraïbe. Ce n'est qu'après 1820 qu'elle envoya ses propres missionnaires aux Indes.

28. Thomas Fanshaw MIDDLETON, A Letter from the Right Reverend the Lord Bishop of Calcutta, addressed to the Reverend Anthony Hamilton, Secretary to the Society for the Propagation of the Gospel in Foreign Parts (18 November 1818), London: S. Brooke, 1819.

29. YATES, op. cit., p. 25.

30. John Poynder (1779-1849), juriste, actionnaire de la Compagnie, et activiste évangélique est l'auteur de plusieurs pamphlets religieux dénonçant en particulier le catholicisme romain. Le sati est une immolation plus ou moins volontaire des épouses sur le bûcher funéraire de leur mari. Claudius Buchanan avait attiré l'attention du public britannique sur le sati comme sur l'infanticide féminin dès 1812 (op. cit., pp. 126-138).

31. Ibid., p. 139.

32. Nancy GARDNER CASSELLS, 'The East India Company's Abbkarry and Pilgrim Taxes. Questions of Public Order and Morality or Revenue?', communication donnée en 2004, $18^{\mathrm{e}}$ congrès de la EASAS : <http://www.sasnet.lu.se/EASASpapers/22NancyGardner.pdf>, p. 58.

33. 'The Church [...] is part of our constitution and forms a chief portion of the respectability of our country ' (BUCHANAN, op. cit., p. 123).

34. Originellement fondées par les baptistes de Serampore à l'initiative de William Ward, elles inspirèrent vite l'action des autres confessions, puisqu'elles permettaient, grâce à des femmes missionnaires, d'atteindre les femmes recluses dans le zenana hindou ou musulman (partie de la demeure familiale réservée aux femmes et aux jeunes enfants).

\section{RÉSUMÉS}

Le réseau d'influence des évangéliques qui s'étend aux diverses Sociétés pour la Promotion du Christianisme comme au Parlement, à la Cité et à la Compagnie des Indes, a joué un rôle considérable dans le développement du pouvoir colonial. Deux aspects essentiels de cette action retiennent l'attention. D'une part, la polémique concernant le libre accès des missionnaires aux territoires de la Compagnie, avec pour corollaire la conduite à observer par celle-ci, en tant que pouvoir tutélaire, envers les religions indigènes et leurs pratiques parfois choquantes pour les actionnaires chrétiens. D'autre part, après 1813 , la création d'un premier diocèse indien de l'Église anglicane, avec des prélats de tendance évangélique marquée, conduisant à des choix déterminants en matière d'éducation et de vie publique, mais sans grandes conséquences pour la christianisation du sous-continent.

The Evangelical interest stretching out to the various Societies for Promoting Christianity as well as the House of Commons, the City and the East India Company played a central part in the development of British colonial power. Two major aspects of Evangelical action are analysed here: firstly, the controversy about the Company territories' accessibility to missionaries and, as a corollary, the behaviour expected from the Company as paramount power towards native religions and their practices, which were sometimes disturbing for the Christian proprietors; secondly, after 1813, the foundation of the first Indian bishopric of the Church of England, with strongly Evangelical bishops, leading to decisive choices in terms of education and public life, but rather inconsequential for the subcontinent's conversion to Christianity. 
AUTEUR

EVELYNE HANQUART-TURNER

Université Paris-Est Créteil et Darwin College, Cambridge 